is as effective as sequential administration in women with endocrine-responsive breast cancer and comment that it might be even more effective than sequential therapy in patients aged $\leq 40$ years.

Original article Del Mastro L et al. (2008) Timing of adjuvant chemotherapy and tamoxifen in women with breast cancer: findings from two consecutive trials of Gruppo Oncologico Nord-Ovest-Mammella Intergruppo (GONO-MIG) Group. Ann Oncol 19: 299-307

\section{Regional lymphadenectomy should be standard for metastasis from melanoma of unknown primary}

Studies examining the prognostic significance of melanoma of unknown primary (MUP) have produced inconsistent results and it is unclear whether patients with MUP require moreaggressive management than patients with known primary melanoma (MKP). Now, Lee et al. have commented that they have conducted the largest ever review of the outcomes of patients with MUP and palpable regional metastasis in a single lymphatic basin, to determine survival rates and prognostic factors.

By examining the clinical records of the 13,000 patients registered on the John Wayne Cancer Institute melanoma database, the researchers identified 1,571 patients with nodal melanoma who underwent regional lymphadenectomy within 3 months of presentation. Among this study group, 262 patients had MUP and 1,309 had MKP. Multivariate analysis revealed that male sex, age $\geq 60$ years, more than one tumorinvolved node, decade of diagnosis, and MKP were all associated with a worse prognosis. The researchers then identified 221 pairs of patients (each comprising one patient with MUP and one patient with MKP) who were matched by the four significant covariates age, sex, nodaltumor burden and decade of diagnosis. The 10-year overall survival rate for patients with MUP was $52 \%$, compared with $36 \%$ for the matched patients with MKP $(P=0.0006$ for trend). In addition, patients with MUP had a longer median overall survival than patients with MKP (165 months vs 34 months; $P=0.0006)$.

The authors conclude that lymphadenectomy should be standard initial treatment for MUP with palpable nodal metastasis limited to a single lymphatic basin; however, they highlight the importance of an accurate staging work-up to rule out the possibility of distant disease.

Original article Lee CC et al. (2008) Improved survival after lymphadenectomy for nodal metastasis from an unknown primary melanoma. J Clin Oncol 26: 535-541

\section{PFS as a surrogate end point for OS in advanced colorectal cancer trials}

Overall survival (OS) is often used as an end point in trials evaluating the efficacy of treatments in colorectal cancer, but measuring this outcome requires prolonged follow-up and the effects of first-line therapies can be influenced by the effects of subsequent therapies.

Buyse et al. retrospectively analyzed data on OS and progression-free survival (PFS) from 13 trials of chemotherapy in advanced colorectal cancer to assess whether PFS could be used as a surrogate for OS in this setting. Ten of these studies were historical trials comparing fluorouracil plus leucovorin with fluorouracil or raltitrexed, whereas three were validation trials of fluorouracil plus leucovorin with or without irinotecan or oxaliplatin.

First, the authors compared OS and PFS from the historical trials (median follow-up 30.4 months) to determine the correlation between these end points. OS and PFS showed a strong correlation (rank correlation coefficient 0.82). The correlation between the effects of treatment on these end points was also high (correlation coefficient 0.99), but it dropped when a particularly influential trial was excluded (correlation coefficient 0.74). The historical trial statistics were then applied to the PFS data in the validation trials to test whether PFS could predict OS. The resulting estimated OS values agreed well with the observed (actual) OS.

The authors conclude that PFS can be used to reliably predict OS in advanced colorectal cancer trials, and that it can, therefore, be used as an alternative primary efficacy end point to avoid the limitations associated with the use of OS.

Original article Buyse M et al. (2007) Progression-free survival is a surrogate for survival in advanced colorectal cancer. J Clin Oncol 25: 5218-5224 